\title{
Evaluating the validities of different DSM-IV-based conceptual constructs of tobacco dependence*
}

\author{
Peter S. Hendricks, Judith J. Prochaska, Gary L. Humfleet \& Sharon M. Hall \\ University of California, San Francisco, CA, USA
}

\begin{abstract}
Aim To compare the concurrent and predictive validities of two subsets of DSM-IV criteria for nicotine dependence (tolerance and withdrawal; withdrawal; difficulty controlling use; and use despite harm) to the concurrent and predictive validity of the full DSM-IV criteria. Design Analysis of baseline and outcome data from three randomized clinical trials of cigarette smoking treatment. Setting San Francisco, California. Participants Two samples of cigarette smokers ( $n=810$ and 322), differing with regard to baseline characteristics and treatment received, derived from three randomized clinical trials. Measurements DSM-IV nicotine dependence criteria were measured at baseline with a computerized version of the Diagnostic Interview Schedule for DSM-IV (DIS-IV). Additional baseline measures included the Fagerström Test of Nicotine Dependence (FTND), number of cigarettes smoked per day, breath carbon monoxide (CO) level, the Minnesota Nicotine Withdrawal Scale (MNWS), the Michigan Nicotine Reinforcement Questionnaire (M-NRQ) and the Profile of Mood States (POMS). Seven-day point-prevalence abstinence was assessed at week 12. Findings Full DSM-IV criteria displayed greater concurrent validity than either of the two subsets of criteria. However, DSM-IV symptoms accounted for only a nominal amount of the variance in baseline smoking-related characteristics and were unrelated to smoking abstinence at week 12. Cigarettes smoked per day was the only significant predictor of abstinence at week 12. Conclusions Although the findings do not provide a compelling alternative to the full set of DSM-IV nicotine dependence criteria, its poor psychometric properties and low predictive power limit its clinical and research utility.
\end{abstract}

Keywords Assessment, diagnosis, DSM, nicotine, smoking, tobacco dependence.

Correspondence to: Peter S. Hendricks, 401 Parnassus Avenue, TRC Box 0984, San Francisco, CA 94143, USA. E-mail: phendricks@lppi.ucsf.edu Submitted 2 October 2007; initial review completed 2 January 2008; final version accepted 26 February 2008

\section{INTRODUCTION}

Measures of tobacco dependence have failed to demonstrate the expected relationships with important smoking-related criteria (see [1]). As a result, researchers have attempted to refine the conceptualization and measurement of this concept. Over the past 6 years several new measures have emerged, including the Hooked on Nicotine Checklist (HONC; [2]), the Cigarette Dependence Scale (CDS; [3]), the Nicotine Dependence Syndrome Scale (NDSS; [4]) and the Wisconsin Inventory of Smoking Dependence Motives (WISDM-68; [5]). It is hoped that these newer tools will reconcile the theoretical importance of the tobacco dependence construct with its modest empirical support.

The criteria for nicotine dependence in the current Diagnostic and Statistical Manual of Mental Disorders (i.e. the DSM-IV; [6]) have also been the focus of recent study (e.g. $[7,8])$, and it has been suggested that these criteria be considered for modification in the upcoming DSM-V (e.g. [9]). Indeed, evidence for the validity of the DSM-IV criteria for nicotine dependence is limited (see [1]). The DSM-IV diagnosis of nicotine dependence requires the presence of at least three of the following seven criteria

*Data in the following manuscript were derived from three clinical trials. Two of these trials are registered with the National Institutes of Health (NIH), and can be accessed at http://clinicaltrials.gov; 'Maintaining Abstinence in Chronic Cigarette Smokers' (identifier: NCT00087880) and 'Maintaining Nonsmoking: Older Smokers' (identifier: NCT00086385). Per NIH regulations, the third trial was not entered into a publicly accessible database because it was funded by a multiple project center grant and hence exempt from the registration requirement. 
during the past year: (i) tolerance; (ii) withdrawal; (iii) using more than intended; (iv) difficulty controlling use; (v) spending a great deal of time (obtaining or using nicotine); (vi) giving up activities; and (vii) use despite harm.

One possible modification of DSM criteria for nicotine dependence was offered recently by Hughes [9], who noted that the same dependence criteria are applied across all drugs of abuse, based on the notion that all substances share common features of problematic use. However, Hughes suggested that not all DSM-IV criteria apply to nicotine dependence. The author proposed that only withdrawal, difficulty controlling use and use despite harm are applicable to nicotine dependence. This assertion was based on observations that these three features are common among cigarette smokers, whereas the remaining four criteria are more common among other drugs of abuse (e.g. [10,11]).

A second potential modification of DSM criteria lies in negative reinforcement models of addiction (e.g. [12,13]) that emphasize the primacy of tolerance and withdrawal (see [14]). In short, these models posit that drug dependence is marked principally by these two features, which are the result of the homeostatic adaptation of the nervous system to chronic drug use. In its definition of substance dependence, the DSM-IV underscores tolerance and withdrawal, and dictates that the presence of these symptoms indicate 'physiological dependence' [6]. Although neither tolerance nor withdrawal are necessary or sufficient for a diagnosis of dependence in the DSM-IV, nicotine dependence criteria based on models of negative reinforcement might focus solely on these two symptoms.

In addition to having immediate implications for the DSM-V, an investigation of nicotine dependence criteria would lend suggestions to similar diagnostic frameworks such as the International Classification of Diseases [15], and would provide insight into the essential features of tobacco dependence. To our knowledge, no previous studies have compared novel sets of DSM items to the complete diagnostic criteria.

The goals of the current investigation were to test the concurrent and predictive validities of the nicotineapplicable criteria offered by Hughes [9] and the negative reinforcement model criteria against the validity of the full DSM-IV criteria for nicotine dependence. Data were drawn from three randomized clinical trials of tobacco dependence. Regression analyses were used to compare the subset criteria offered by Hughes and the negative reinforcement model to the full DSM-IV criteria in predicting baseline smoking-related characteristics (e.g. cigarettes smoked per day) and treatment outcome (i.e. smoking status). If either of the two subsets of specific criteria encapsulates the key features of tobacco dependence, then these criteria should explain a significant and robust amount of the variance in baseline smokingrelated characteristics and treatment outcome, and the remaining DSM-IV criteria should provide for a marginal addition in explained variance. If neither subset encompasses the core characteristics of tobacco dependence, then we should find that the addition of the remaining DSM-IV criteria provide for a marked increase in explained variance.

\section{METHODS}

\section{Source data}

Data were drawn from three randomized clinical trials of cigarette smoking treatment conducted in San Francisco, $\mathrm{CA}$, from which we formed two samples: 'chronic smokers' and 'depressed smokers'. Data from these two samples were analyzed separately to provide for replication of the findings among different groups of smokers and varying treatment contexts.

\section{Sample 1: chronic smokers}

Data from two clinical trials to 12 weeks of treatment were merged to form this sample $(n=810)$. Both trials were designed to test interventions for chronic smoking behavior, and had similar interventions across treatment conditions during the first 12 weeks of treatment. Although inclusion criteria for the two trials differed to a degree, participant characteristics across trials were largely comparable. The two samples were combined to provide for increased power to detect relationships among variables of interest.

Participants from both trials were recruited through direct provider referral, flyers, advertisements, public service announcements and outreach to community organizations. Inclusion criteria for trial $1(n=407)$ were smoking at least 10 cigarettes per day and smoking within 30 minutes of awakening. Inclusion criteria for trial $2(n=403)$ were smoking at least 10 cigarettes per day and being at least 50 years old. Intention to quit smoking was an inclusion criterion for both trials. Exclusion criteria for both trials were inability to speak English, use of psychiatric drugs, presence of a condition that contraindicated use of pharmacological treatments or presence of conditions that might interfere with compliance with protocol (e.g. severe alcohol abuse) or greatly complicate treatment (e.g. acutely life-threatening disease). An additional exclusion criterion of trial 1 was age younger than 18 years.

Participants from both trials who met the screening criteria during a telephone interview were provided with an overview of the program and invited to the project offices to complete a pre-treatment baseline assessment and screening physical examination. Upon completion of 
the baseline assessment, participants were assigned randomly to experimental conditions. Interventions for both trials to 12 weeks consisted of 12 weeks of bupropion, 10 weeks of nicotine replacement therapy (NRT) and five group counseling sessions. Participants initiated the use of $300 \mathrm{mg}$ per day of buproprion during week 1 . Trial 1 participants received a 10-week supply of the transdermal nicotine patch, whereas participants in trial 2 received a 10-week supply of the nicotine gum. In both trials participants initiated the use of NRT on their quit date, which occurred during week 2. Intervention components of the group counseling sessions included psychoeducational didactics, and the development of personalized quit plans centered on cognitive and behavior coping skills.

\section{Sample 2: depressed smokers}

Data from one clinical trial to 12 weeks of treatment formed this sample $(n=322)$. This randomized trial was designed to investigate treatment for smoking among currently depressed mental health out-patients. Study recruitment, design and intervention components have been described in detail elsewhere [16-18]. Briefly, patients diagnosed with current unipolar depression, over 18 years of age, who smoked at least one cigarette per day, and were in active mental health treatment at one of the participating clinics were recruited. Intention to quit smoking was not a prerequisite for study participation. Exclusion criteria were inability to speak English, history of bipolar disorder or contraindications to use of NRT or bupropion.

Participants in the control condition $(n=159)$ received smoking cessation referrals and a self-help pamphlet. At baseline, participants in the experimental condition ( $n=163$ ) completed a computer-delivered expert system intervention based on the Stages of Change model and received individualized feedback designed to motivate smokers to quit. Treatment participants interested in quitting also could receive cessation treatment consisting of the nicotine patch and six 30-minute sessions of individual counseling provided over the course of 8 weeks focused on mood monitoring and management, behavioral relapse prevention strategies and relaxation and social support skills [19].

\section{Participant characteristics}

The chronic smoker sample was $60 \%$ male with a mean age of 48.7 years [standard deviation $(\mathrm{SD})=11.3$ ]. Of this sample, $77 \%$ were white, $8 \%$ were black/African American, 5\% were Asian/Pacific Islander, 9\% were multi-racial, $1 \%$ were American Indian/Alaska Native and $6 \%$ were Hispanic/Latino (assessed across all racial categories). The sample averaged 30.3 years $(\mathrm{SD}=11.9)$ of smoking; 19.8 cigarettes smoked per day $(\mathrm{SD}=8.1$ ), indicating a heavy smoking rate; an FTND score of 4.9 $(\mathrm{SD}=2.0)$, indicating a moderate level of nicotine dependence; and a breath carbon monoxide $(\mathrm{CO})$ reading of 21.49 parts per million (p.p.m.) $(\mathrm{SD}=10.7)$, indicating recent use of cigarettes.

The depressed smoker sample was 30\% male with a mean age of 41.8 years $(\mathrm{SD}=12.5)$. Of this sample, $69 \%$ were white, $10 \%$ were black/African American, $2 \%$ were Asian/Pacific Islander, 8\% were'other', 4\% were multiracial and $7 \%$ were Hispanic/Latino (assessed as a distinct racial category). The sample averaged 24.3 years $(\mathrm{SD}=12.8)$ of smoking, 15.5 cigarettes smoked per day $(\mathrm{SD}=10.1)$, indicating a moderate smoking rate; an FTND score of $4.0(\mathrm{SD}=2.5)$, indicating a moderate level of nicotine dependence; and a $\mathrm{CO}$ reading of 15.35 p.p.m. $(\mathrm{SD}=10.05)$, indicating recent use of cigarettes.

\section{Measures}

Diagnostic interview schedule for DSM-IV (DIS-IV; [20])

A computerized version of DIS-IV was administered at baseline to both the chronic smoker and depressed smoker samples. Each of the seven DSM-IV nicotine dependence criteria was assessed as being either present or absent in the past year with this instrument. Consistent with the DSM-IV, participants were diagnosed as nicotine-dependent if they reported experiencing three or more of the seven nicotine dependence symptoms in the past year. Participants were diagnosed with nicotine withdrawal if they endorsed the characteristic nicotine withdrawal syndrome or if they reported using nicotine to relieve or avoid withdrawal symptoms.

\section{Fagerström Test of Nicotine Dependence (FTND; [21])}

The FTND, which measures physical tobacco dependence, was administered at baseline to both samples. This measure was incorporated in concurrent validation analyses because it is the most widely used measure of dependence, and data support its validity (see [1]).

\section{Smoking Information Questionnaire (SIQ) and breath CO}

The SIQ assessed number of cigarettes smoked per day, while CO provided biochemical verification of cigarette use. These measures were administered at baseline to both samples. They were included in the concurrent validation analyses because the degree of cigarette use (i.e. smoking heaviness) is considered central to tobacco dependence (e.g. [22]).

\section{Minnesota Nicotine Withdrawal Scale (MNWS; [23])}

The MNWS, an eight-item questionnaire that measures each symptom of withdrawal over the past 24 hours, was 
administered at baseline to the chronic smoker sample. The MNWS has favorable psychometric properties and compares well with other measures of tobacco withdrawal [24]. This measure was used in concurrent validation analyses because withdrawal is believed to be a fundamental criterion of tobacco dependence (e.g. [25]).

\section{Michigan Nicotine Reinforcement Questionnaire} (M-NRQ; [26])

The M-NRQ, a self-rating scale that measures positivereinforcement smoking (Positive Reinforcement Scale; PRS) and negative-reinforcement smoking (Negative Reinforcement Scale; NRS), was administered to the chronic smoker sample at baseline. This measure was included in concurrent validation analyses because its scales have been shown to be associated with number of variables relevant to cigarette use, including nicotine dependence (as measured by an earlier version of the FTND).

Profile of Mood States (POMS; [27])

The POMS, which provides six subscale scores and a Total Mood Disturbance score (TMD), was administered at baseline to the chronic smoker sample. POMS scores have been shown to be sensitive to a variety of drug and psychotherapeutic interventions, as well as smoking relapse (e.g. [19]). The POMS was used in concurrent validation analyses because the regulation of negative affect is proposed to be the primary motivational factor in cigarette and other drug use [28].

\section{Point-prevalence abstinence}

Participants' self-reported smoking status in the past week, verified with the use of CO, was assessed at week 12 in both samples. This measure was used in predictive validity analyses because the prediction of treatment outcome is considered a 'gold standard' for tobacco dependence measures (e.g. [9]).

\section{Analysis strategy}

Three regression models were examined: (i) the "nicotineapplicable' model suggested by Hughes [9], consisting of withdrawal, difficulty controlling use and use despite harm as the independent variables; (ii) the 'negativereinforcement' model, consisting of tolerance and withdrawal as the independent variables; and (iii) the 'full' model, containing each of the seven DSM-IV criteria as the independent variables. In each model, each of the criteria were coded as $0=$ 'absent,' $1=$ 'present.' Both the nicotine-applicable model and the negativereinforcement model were nested in the full model.
Data analyses were conducted in two phases. First, we tested whether the full model provided for a significant increment in the proportion of variance explained in baseline smoking-related characteristics above and beyond either the nicotine-applicable model or the negative-reinforcement model using linear regression analyses. Analyses were conducted as follows: (i) both the nicotine-applicable model and the negativereinforcement model were estimated; (ii) the remaining DSM-IV items missing from each model were added to the nicotine-applicable model and the negativereinforcement model; thus, each resulting in the same full model; (iii) the full model was estimated, and the increment in $R^{2}$ upon the addition of the remaining items to the nicotine-applicable model and the negativereinforcement model was tested. These analyses represented the tests of concurrent validity of the three models.

Next, we tested if the full model was a more robust predictor of smoking status at week 12 than either the nicotine-applicable model or the negative-reinforcement model using logistic regression analyses. Analyses were conducted as follows: (i) both the nicotine-applicable model and the negative-reinforcement model were estimated; (ii) the remaining DSM-IV items missing from each model were added to the nicotine-applicable model and negative-reinforcement model; thus, each resulting in the same full model; (iii) the full model was estimated and the change in goodness-of-fit upon the addition of the remaining items to nicotine-applicable model and the negative-reinforcement model was tested. These analyses represented the tests of predictive validity of the three models.

\section{RESULTS}

\section{DSM-IV criteria}

Table 1 displays the percentage of participants in both treatment groups who endorsed each DSM-IV criterion. Among chronic smokers, internal consistency of the nicotine-applicable (Cronbach's alpha $=0.33$ ), negativereinforcement (Cronbach's alpha $=0.37$ ) and full (Cronbach's alpha $=0.54$ ) model items were below conventional standards for clinical use (e.g. [29]). Among depressed smokers, internal consistency of the nicotine-applicable (Cronbach's alpha $=0.25$ ), negative-reinforcement (Cronbach's alpha $=0.39$ ) and full (Cronbach's alpha $=0.51$ ) model items also were below conventional standards. These results suggest that validity analyses may have been compromised by the models' poor reliability. Seventy-one percent of chronic smokers and $69 \%$ of depressed smokers met DSM-IV criteria for current nicotine dependence. 
Table 1 Frequency of endorsed DSM-IV criteria by treatment group.

\begin{tabular}{lll}
\hline & \multicolumn{2}{l}{ Treatment group } \\
\cline { 2 - 3 } Criterion & Chronic smokers (\%) & Depressed smokers (\%) \\
\hline Tolerance & 51 & 39 \\
Withdrawal & 56 & 57 \\
Using more than intended & 75 & 83 \\
Difficulty controlling use & 98 & 94 \\
Spending a great deal of time & 43 & 43 \\
Giving up activities & 21 & 23 \\
Use despite harm & 53 & 54 \\
\hline
\end{tabular}

Table 2 Prediction of baseline smoking characteristics: chronic smokers.

\begin{tabular}{lccccc}
\hline & Nicotine-applicable $R^{2}$ & Negative-reinforcement $R^{2}$ & Full $R^{2}$ & Nicotine-applicable $\Delta R^{2}$ & Negative-reinforcement $\Delta R^{2}$ \\
\hline FTND & $0.03(<0.0001)$ & $0.04(<0.0001)$ & $0.07(<0.0001)$ & $0.04(<0.0001)$ & $0.03(<0.0001)$ \\
CPD & $<0.01(0.37)$ & $0.01(0.02)$ & $0.03(<0.0001)$ & $0.03(<0.0001)$ & $0.02(0.0002)$ \\
CO & $<0.01(0.87)$ & $<0.01(0.07)$ & $<0.01(0.16)$ & - & - \\
MNWS & $0.05(<0.0001)$ & $0.03(0.0008)$ & $0.08(<0.0001)$ & $0.03(0.008)$ & $0.05(0.0003)$ \\
PRS & $<0.01(0.09)$ & $<0.01(0.72)$ & $<0.01(0.37)$ & - & - \\
NRS & $0.21(<0.0001)$ & $0.21(<0.0001)$ & $0.26(<0.0001)$ & $0.05(<0.0001)$ & $0.05(<0.0001)$ \\
TMD & $0.04(<0.0001)$ & $0.02(0.0004)$ & $0.05(<0.0001)$ & $0.01(0.009)$ & $0.03(<0.0001)$
\end{tabular}

$R^{2}$ : adjusted $R^{2} \cdot R^{2}$ statistics with $P$-values of less than 0.05 are presented in bold type; $P$-values are presented in parentheses. Nicotine-applicable $\Delta R^{2}$ : change in adjusted $R^{2}$ from nicotine-applicable to full model. Negative-reinforcement $\Delta \mathrm{R}^{2}$ : change in adjusted $R^{2}$ from negative-reinforcement to full model. FTND: Fagerström Test of Nicotine Dependence; CPD: cigarettes smoked per day; CO: breath carbon monoxide; MNWS: Minnesota Nicotine Withdrawal Scale; PRS: Positive Reinforcement Scale of the Michigan Nicotine Reinforcement Questionnaire; NRS: Negative Reinforcement Scale of the Michigan Nicotine Reinforcement Questionnaire; TMD: Total Mood Disturbance scale of the Profile of Mood States.

Table 3 Prediction of baseline smoking characteristics: depressed smokers.

\begin{tabular}{llllll}
\hline & Nicotine-applicable $R^{2}$ & Negative-reinforcement $R^{2}$ & Full $R^{2}$ & Nicotine-applicable $\Delta R^{2}$ & Negative-reinforcement $\Delta R^{2}$ \\
\hline FTND & $0.06(<0.0001)$ & $00.09(<0.0001)$ & $0.15(<0.0001)$ & $0.09(<0.0001)$ & $0.06(0.0001)$ \\
CPD & $<0.01(0.19)$ & $00.02(0.02)$ & $0.07(0.0001)$ & $0.07(<0.0001)$ & $0.05(0.0007)$ \\
CO & $<0.01(0.63)$ & $00.02(0.02)$ & $0.03(0.02)$ & $0.03(0.007)$ & $0.01(0.16)$ \\
\hline
\end{tabular}

$R^{2}$ : adjusted $R^{2} . R^{2}$ statistics with $P$-values of less than 0.05 are presented in bold type; $P$-values are presented in parentheses. Nicotine-applicable $\Delta \mathrm{R}^{2}$ : change in adjusted $R^{2}$ from nicotine-applicable to full model. Negative-reinforcement $\Delta R^{2}$ : change in adjusted $R^{2}$ from negative-reinforcement to full model. FTND: Fagerström Test of Nicotine Dependence; CPD: cigarettes smoked per day; CO = breath carbon monoxide.

\section{Concurrent validation}

Tables 2 and 3 shows the results from regression analyses for chronic smokers and depressed smokers, respectively. With rare exception, the full model explained a significantly greater amount of variance than both the nicotine-applicable model and the negativereinforcement model in smoking-related characteristics. However, the full model accounted for very little of the variance in these variables.

\section{Concurrent validity of specific DSM items}

Because neither the nicotine-applicable model nor negative-reinforcement model demonstrated superior concurrent validity to the full DSM-IV criteria, the concurrent validity of each DSM-IV criterion was investigated. The purpose of this analysis was to identify those items that appeared to differentiate most clearly the less dependent from the more dependent smokers. Only those baseline smoking-related characteristics that the full model had predicted significantly were evaluated in this portion of the analysis.

Tables 4 and 5 display significant predictors of smoking-related baseline characteristics among chronic and depressed smokers, respectively. As displayed in the tables, DSM-IV criteria predicted a range of smokingrelated variables. Among the most consistent predictors was giving up activities (a significant predictor of five of 
Table 4 Predictors of baseline smoking characteristics among DSM-IV criteria: chronic smokers.

\begin{tabular}{|c|c|c|c|c|c|}
\hline & FTND & $C P D$ & $M W S$ & NRS & $T M D$ \\
\hline Tolerance & $0.47(0.003)$ & $1.19(0.06)$ & $0.17(0.77)$ & $1.48(0.001)$ & $2.28(0.31)$ \\
\hline Withdrawal & $0.27(0.08)$ & $-0.38(0.54)$ & $1.66(0.004)$ & $3.42(<0.0001)$ & $5.02(0.027)$ \\
\hline Using more than intended & $0.16(0.38)$ & $0.77(0.28)$ & $1.38(0.04)$ & $0.36(0.48)$ & $5.12(0.049)$ \\
\hline Difficulty controlling use & $-0.25(0.68)$ & $-3.04(0.22)$ & $-9.84(0.002)$ & $-6.97(0.004)$ & $-27.17(0.003)$ \\
\hline Spending a great deal of time & $0.36(0.02)$ & $2.53(<0.0001)$ & $0.87(0.12)$ & $0.91(0.037)$ & $3.01(0.18)$ \\
\hline Giving up activities & $0.68(0.0002)$ & $1.48(0.047)$ & $1.53(0.016)$ & $1.24(0.01)$ & $5.47(0.04)$ \\
\hline Use despite harm & $0.30(0.053)$ & $0.04(0.94)$ & $0.37(0.51)$ & $1.11(0.01)$ & $5.97(0.008)$ \\
\hline
\end{tabular}

Reported statistic is standardized regression coefficient. Standardized regression coefficients with $P$-values of less than 0.05 are presented in bold type; $P$-values are presented in parentheses. FTND: Fagerström Test of Nicotine Dependence; CPD: cigarettes smoked per day; MNWS: Minnesota Nicotine Withdrawal Scale; NRS: Negative Reinforcement Scale of the Michigan Nicotine Reinforcement Questionnaire; TMD: Total Mood Disturbance scale of the Profile of Mood States.

Table 5 Predictors of baseline smoking characteristics among DSM-IV criteria: depressed smokers.

\begin{tabular}{lcrr}
\hline & FTND & CPD & CO \\
\hline Tolerance & $0.68(0.02)$ & $1.38(0.25)$ & $2.8(0.02)$ \\
Withdrawal & $0.77(0.006)$ & $0.76(0.51)$ & $-0.39(0.74)$ \\
Using more than intended & $-0.42(0.24)$ & $-1.11(0.47)$ & $1.29(0.40)$ \\
Difficulty controlling use & $0.45(0.44)$ & $-1.53(0.52)$ & $1.99(0.42)$ \\
Spending a great deal of time & $0.60(0.03)$ & $3.24(0.006)$ & $0.41(0.73)$ \\
Giving up activities & $1.38(<0.0001)$ & $4.62(0.0009)$ & $3.14(0.03)$ \\
Use despite harm & $0.054(0.84)$ & $-0.65(0.57)$ & $-1.66(0.16)$ \\
& & &
\end{tabular}

Reported statistic is standardized regression coefficient. Standardized regression coefficients with $P$-values of less than 0.05 are presented in bold type; $P$-values are presented in parentheses. FTND: Fagerström Test of Nicotine Dependence; CPD: cigarettes smoked per day; CO: breath carbon monoxide.

five variables among chronic smokers and three of three variables among depressed smokers) and spending a great deal of time (a significant predictor of three of five variables among chronic smokers and two of three variables among depressed smokers). Finally, although chronic smokers who did not endorse difficulty controlling use were more likely to report higher withdrawal, negative reinforcement smoking, and mood disturbance, the lack of variance in response to this item suggests that this finding may have been anomalous.

\section{Predictive validity}

None of the three models predicted the likelihood of smoking abstinence at week 12 for either chronic smokers (all Ps $>0.50$ ) or depressed smokers (all Ps $>0.30)$. Because the prediction of treatment outcome (i.e. smoking status) is considered one of the most important tests of validation for measures of tobacco dependence (e.g. [9]), the predictive validities of three alternative measures that have demonstrated significant validity in other studies of tobacco dependence also were examined. These were: FTND scores (e.g. [30,31]), time to first cigarette of the day [32] and cigarettes smoked per day (e.g. [33]).
Among chronic smokers, FTND scores $($ Wald $=14.66$, $\beta=-0.14, P=0.0001)$, time to first cigarette of the day (Wald $=11.66, \beta=-0.27, P=0.0006)$ and cigarettes per day (Wald $=13.32, \beta=-0.03, P=0.0003$ ) each significantly predicted probability of smoking abstinence at week 12. To determine the strongest predictor of smoking abstinence among these three variables, FTND, time to first cigarette and cigarettes smoked per day were entered into a simultaneous logistic regression. Results indicated that FTND (Wald $=0.74, \beta=-0.05, P=0.38$ ) and time to first cigarette (Wald $=1.24, \beta=-0.13, P=0.26$ ) were no longer related to likelihood of smoking abstinence at week 12 , whereas higher daily cigarette consumption at baseline (Wald $=4.24, \beta=-0.02, P=0.04$ ) continued to be associated with a lower probability of smoking abstinence at week 12 .

Among depressed smokers in the control condition, FTND scores $($ Wald $=1.76, \beta=-0.15, P=0.18)$ and time to first cigarette (Wald $=0.30, \beta=-0.13, P=0.58$ ) were unrelated to smoking abstinence probability at week 12 . However, higher daily cigarette consumption (Wald $=4.75, \beta=-0.08, P=0.03$ ) was associated with a lower probability of smoking abstinence at this time. Among depressed smokers in the experimental condition, FTND scores $($ Wald $=2.15, \beta=-0.15, P=0.14)$, time to 
first cigarette (Wald $=2.27, \beta=-0.32, P=0.13)$ and cigarettes smoked per day (Wald $=0.81, \beta=-0.02$, $P=0.37)$ were each unrelated to smoking abstinence likelihood at week 12 .

\section{DISCUSSION}

Our findings support the incremental validity of the full DSM-IV criteria over both of the subsets of items explored in the current investigation. Accordingly, our results offer no decisive alternative to the full set of DSM-IV nicotine dependence criteria. However, the full DSM criteria accounted for only a very modest percentage of variance in smoking-related characteristics, and failed to predict treatment outcome. Furthermore, DSM-IV items demonstrated internal consistency below standards for clinical use. These findings are consistent with previous research (see [1]) and suggest that the DSM-IV items, as they are currently constructed, may not be well suited for most clinical or research purposes. Although there may be clinical utility in providing a diagnosis of dependence for third-party reimbursement, the primary strength of the DSM-IV and DSM-type measures (e.g. [15]) may lie in their ability to provide a somewhat basic description of tobacco dependence.

Cigarettes smoked per day was the strongest predictor of treatment outcome, outperforming FTND scores and time to first cigarette. This finding is consistent with past research [33], and suggests that while cigarette consumption may represent a rather unsophisticated indicator of dependence it may nevertheless be a more sensitive measure than some of the most widely used indices.

The relatively poor psychometric properties of DSM-IV nicotine dependence criteria do not necessarily indicate that these criteria are not valid indicators of the dependence construct. Rather, the chief shortcoming of DSM-IV items may be the manner in which they are assessed. The dichotomous scoring of each item may mask the true range of symptom severity and reduce the power of the DSM-IV (see [34,35]), and because the DSM-IV uses single items to assess symptom domains, item-specific error may be exaggerated (see [29]). These limitations may attenuate the reliability and validity of the measure. Indeed, the CDS [3], which incorporates some of the DSM-IV items and measures them in a continuous manner, demonstrates good reliability.

Review of predictors of smoking characteristics among DSM-IV criteria points to which items may be especially useful at signaling higher levels of dependence if assessed dimensionally. While all items predicted baseline smoking variables to a degree, giving up activities and spending a great deal of time appear to carry relatively unique significance. Notably, these items were the two least frequently endorsed of all DSM-IV criteria. One potential explanation for this finding is that the more frequently endorsed items reflect processes that occur relatively early in the dependence process, whereas those items that are endorsed relatively infrequently reflect phenomena that occur in the later, more severe, stages of addiction. Consistent with this interpretation, past research using item response modeling found that giving up activities was endorsed least frequently, yet was associated with the highest levels of involvement with cigarettes among a large population of smokers [36]. This suggests that the upcoming DSM-V should retain those criteria that appear to be more characteristic of other forms of drug dependence. Note, however, that the current results do not address the question of whether dependence manifests itself similarly across substances. In fact, recent research indicates that the DSM-IV criteria measure different levels of dependence across different types of illicit drugs [37]. Thus, as suggested by Hughes [9], tobacco dependence may manifest itself differently than other forms of substance dependence.

\section{Limitations}

There were limitations to this investigation that should be acknowledged. The computerized DIS may not be the ideal method of assessing DSM-IV nicotine dependence symptoms. It is possible that an alternate approach, such as expert interview, would yield different results. Furthermore, the clinical nature of the study's samples limits the generalizability of the findings. Specifically, the participants in the current study may have been more tobaccodependent than the population of cigarette smokers. This could explain the lack of variance in some of the item responses (e.g. difficulty controlling use), which likely reduced the reliability of the measure, as well affected its relations with other variables (see [29]). Thus, the continued assessment of DSM-IV criteria may prove worthwhile.

\section{Recommendations and future directions}

Our findings add to a body of literature indicating that the DSM-IV is an ill-suited index of tobacco dependence. Given the general acceptance and wide use of the DSM, it would be impractical to recommend that the tool be abandoned. How, then, can the validity of DSM-IV nicotine dependence criteria be improved?

Perhaps one approach lies in contemporary theories that regard dependence as a continuous construct with multiple degrees of severity (see [38]). This view is somewhat incompatible with the psychiatric diagnostic model upon which the DSM-IV and similar measures (e.g. [15]) are based. However, the possible benefit to the DSM of assessing each nicotine dependence criterion on a continuum should be explored. Moreover, our results 
suggest that a continuous measure of consumption (e.g. cigarettes smoked per day) may have its place in such a measurement system. Nevertheless, until the DSM shows improvement, clinicians and researchers alike should continue to incorporate multiple, alternative measures of tobacco dependence.

\section{Acknowledgements}

This study was supported by NIDA grants R01 DA15732, R01 DA02538, P50 DA09253, T32 DA007250, K23 DA018691 and K05 DA016752 and the State of California Tobacco-Related Disease Research Program grant 13KT-0152. The authors thank Dr Kevin Delucchi for his helpful comments.

\section{References}

1. Piper M. E., McCarthy D. E., Baker T. B. Assessing tobacco dependence: a guide to measure evaluation and selection. Nicotine Tob Res 2006; 8: 339-51.

2. DiFranza J. R., Savageau J. A., Fletcher K., Ockene J. K., Rigotti N. A., McNeill A. D. et al. Measuring the loss of autonomy over nicotine use in adolescents: the Development and Assessment of Nicotine Dependence in Youths (DANDY) study. Arch Pediatr Adolesc Med 2002; 23: 397403.

3. Etter J. F., Le Houezec J., Perneger T. V. A self-administered questionnaire to measure dependence on cigarettes: the Cigarette Dependence Scale. Neuropsychopharmacology 2003; 28: 359-70.

4. Shiffman S., Waters A. J., Hickcox M. The Nicotine Dependence Syndrome Scale: a multidimensional measure of nicotine dependence. Nicotine Tob Res 2004; 6: 327-49.

5. Piper M. E., Piasecki T. M., Federman E. B., Bolt D. M., Smith S. S., Fiore M. C. et al. A multiple motives approach to tobacco dependence: the Wisconsin Inventory of Smoking Dependence Motives (WISDM-68). J Consult Clin Psychol 2004; 72: 139-54.

6. American Psychiatric Association. Diagnostic and Statistical Manual of Mental Disorders, 4th edn. Washington, DC: American Psychiatric Association; 1994.

7. Muthen B., Asparouhov T. Item response mixture modeling: application to tobacco dependence criteria. Addict Behav 2006; 31: 1050-66.

8. Lessov C. N., Martin N. G., Statham D. J., Todorov A. A., Slutske W. S., Bucholz K. K. et al. Defining nicotine dependence for genetic research: evidence from Australian twins. Psychol Med 2004; 34: 865-79.

9. Hughes J. R. Should criteria for drug dependence differ across drugs? Addiction 2006; 101: 134-41.

10. Breslau N., Kilbey M. M., Andreski P. DSM-III-R nicotine dependence in young adults: prevalence, correlates, and associated psychiatric disorders. Addiction 1994; 89: 74354.

11. John U., Meyer C., Hapke U., Rumpf H. J. Nicotine dependence and lifetime amount of smoking in a population sample. Eur J Public Health 2004; 14: 182-5.

12. Wikler A. Dynamics of drug dependence: implications of a conditioning theory for research and treatment. Arch Gen Psychiatry 1973; 28: 611-16.
13. Siegel S. Drug anticipation and drug addiction. The 1998 H. David Archibald Lecture. Addiction 1999; 94: 1113-24.

14. Eissenberg T. Measuring the emergence of tobacco dependence: the contribution of negative reinforcement models. Addiction 2004; 99: 5-29.

15. World Health Organization. ICD-10: International Statistical Classification of Diseases and Related Health Problems, 10th revision. Geneva: World Health Organization; 1992.

16. Prochaska J. J., Hall S. M., Tsoh J. Y., Eisendrath S., Rossi J. S., Redding C. A. et al. Treating tobacco dependence in clinically depressed smokers: effect of smoking cessation on mental health functioning. Am J Public Health 2007; 97: $12-15$.

17. Hall S. M., Tsoh J. Y., Prochaska J. J., Eisendrath S., Rossi J. S., Redding C. A. et al. Treatment for cigarette smoking among depressed mental health outpatients: a randomized clinical trial. Am J Public Health 2006; 96: 1808-14.

18. Prochaska J. J., Rossi J. S., Redding C. A., Rosen A. B., Tsoh J. Y., Humfleet G. L. et al. Depressed smokers and stage of change: implications for treatment interventions. Drug Alcohol Depend 2004; 76: 143-51.

19. Hall S. M., Munoz R. F., Reus V. I. Cognitive-behavioral intervention increases abstinence rates for depressivehistory smokers. J Consult Clin Psychol 1994; 62: 1416.

20. Robins L. N., Cottler L., Bucholz K., Compton W. Diagnostic Interview Schedule for DSM-IV (DIS-IV). St Louis, MO: Department of Psychiatry, Washington University School of Medicine; 1995.

21. Heatherton T. F., Kozlowski L. T., Frecker R. C., Fagerström K. O. The Fagerström Test for Nicotine Dependence: a revision of the Fagerström Tolerance Questionnaire. Br J Addict 1991; 86: 1119-27.

22. Baker T. B., Brandon T. H., Chassin L. Motivational influences on cigarette smoking. Annu Rev Psychol 2004; 55: 463-91.

23. Hughes J. R., Hatsukami D. K. Signs and symptoms of tobacco withdrawal. Arch Gen Psychiatry 1986; 43: 28994.

24. Etter J. F., Hughes J. R. A comparison of the psychometric properties of three cigarette withdrawal scales. Addiction 2006; 101: 362-72.

25. Hendricks P. S., Ditre J. W., Drobes D. J., Brandon T. H. The early time course of smoking withdrawal effects. Psychopharmacology 2006; 187: 385-96.

26. Pomerleau O. F., Fagerström K. O., Marks J. L., Tate J. C., Pomerleau C. S. Development and validation of a self-rating scale for positive- and negative-reinforcement smoking: the Michigan Nicotine Reinforcement Questionnaire. Nicotine Tob Res 2003; 5: 711-18.

27. McNair D. M., Lorr M., Droppleman L. F. Manual for the Profile of Mood States. San Diego, CA: Educational and Instructional Testing Service; 1981.

28. Baker T. B., Piper M. E., McCarthy D. E., Majeskie M. R., Fiore M. C. Addiction motivation reformulated: an affective processing model of negative reinforcement. Psychol Rev 2004; 111: 33-51.

29. Nunnally J. C., Bernstein I. H. Psychometric Theory, 3rd edn. New York: McGraw-Hill; 1994.

30. Patten C. A., Martin J. E., Calfas K. J., Lento J., Wolter T. D. Behavioral treatment for smokers with a history of alcoholism: predictors of successful outcome. J Consult Clin Psychol 2001; 69: 796-801. 
31. Westman E. C., Behm F. M., Simel D. L., Rose J. E. Smoking behavior on the first day of a quit attempt predicts longterm abstinence. Arch Inten Med 1997; 157: 335-40.

32. Fagerström K. O. Time to first cigarette: the best single indicator of tobacco dependence? Monaldi Arch Chest Dis 2003; 59: 95-8.

33. Breslau N., Johnson E. O. Predicting smoking cessation and major depression in nicotine-dependent smokers. Am J Public Health 2000; 90: 1122-7.

34. Streiner D. L. Breaking up is hard to do: the heartbreak of dichotomizing continuous data. Can J Psychiatry 2002; 47: 262-6.

35. MacCallum R. C., Zhang S., Preacher K. J., Rucker D. D. On the practice of dichotomization of quantitative variables. Psychol Methods 2002; 7: 19-40.
36. Strong D. R., Kahler C. W., Ramsey S. E., Brown R. A. Finding order in the DSM-IV nicotine dependence syndrome: a Rasch analysis. Drug Alcohol Depend 2003; 72: 151-62.

37. Gillespie N. A., Neale M. C., Prescott C. A., Aggen S. H., Kendler K. S. Factor and item-response analysis DSM-IV criteria for abuse of and dependence on cannabis, cocaine, hallucinogens, sedatives, stimulants, and opioids. Addiction 2007; 102: 920-30.

38. Tiffany S. T., Conklin C. A., Shiffman S., Clayton R. R. What can dependence theories tell us about assessing the emergence of tobacco dependence? Addiction 2004; 99: 78-86. 